\title{
Competition and Adaptation in an Internet Evolution Model
}

\author{
M. Ángeles Serrano, Marián Boguñá, and Albert Díaz-Guilera \\ Departament de Física Fonamental, Universitat de Barcelona, Martí i Franquès 1, 08028 Barcelona, Spain
} (Received 12 July 2004; published 24 January 2005)

\begin{abstract}
We model the evolution of the Internet at the autonomous system level as a process of competition for users and adaptation of bandwidth capability. From a weighted network formalism, where both nodes and links are weighted, we find the exponent of the degree distribution as a simple function of the growth rates of the number of autonomous systems and connections in the Internet, both empirically measurable quantities. Our approach also accounts for a high level of clustering as well as degree-degree correlations, both with the same hierarchical structure present in the real Internet. Further, it also highlights the interplay between bandwidth, connectivity, and traffic of the network.
\end{abstract}

DOI: 10.1103/PhysRevLett.94.038701

A statistical physics approach to Internet modeling will be successful only if its large-scale properties can be explained and predicted on the basis of the interactions between basic units at the microscopic level $[1,2]$. Dynamical evolution rules acting at the local scale would then determine the behavior and the emergent structural properties of the whole Internet, which self-organizes under an absolute lack of centralized control. This approach is at the core of a set of recent network models focusing on evolution, which recognize growth as one of the key mechanisms on network formation, along with preferential attachment or other utility rules [3-10]. However, most of these models consider that the evolution of the Internet is ruled by mere topological quantities, ignoring the heterogeneity of nodes and the fact that connections between nodes are weighted. In this Letter, we present a new growing network model for the Internet evolution at the autonomous system (AS) level in which both, nodes and links, are weighted. The AS level represents a coarse grained description of the Internet, in which ASs are defined as independently administered domains which autonomously determine internal communications and routing policies [1]. As a first approximation we can assign each AS to an Internet service provider. Under the mechanisms of exponential growth, competition, and adaptation, the model reproduces the topological properties observed in the AS level maps of the Internet, namely, (i) a scale-free distribution of the number of connections or degree-of vertices $k_{i}$, of the form $P(k) \propto k^{-\gamma}, 2.1 \leq$ $\gamma<2.5$, (ii) high clustering coefficient $c_{k}$-defined as the ratio between the number of connected neighbors of a node of degree $k$ and the maximum possible value averaged for all nodes of degree $k$-that shows a hierarchical structure, and, finally, (iii) disassortative degree-degree correlations, quantified by means of the average nearest neighbors degree of nodes of degree $k, \bar{k}_{n n}(k)[11,12]$. In addition, our model provides deeper insights on the Internet evolution while allowing predictions on the weight (bandwidth) of connections between ASs and the weight (number of users) of ASs.
PACS numbers: 89.20.Hh, 05.70.Ln, 87.23.Ge, 89.75.Hc

We start our analysis by looking at the growth of the Internet during the last few years. We focus on the temporal evolution of the number of hosts present in the Internet [13] as compared to the number of distinct ASs and the total number of connections among them. We have reanalyzed AS maps collected by the Oregon route-views project which has recorded the Internet topology at the AS level since November 1997 [14]. Let $W(t), N(t)$, and $E(t)$ be the total number of hosts (we assume that number of hosts is equivalent to number of users), number of ASs, and edges among ASs at time $t$, respectively. Figure 1 shows empirical measurements for these quantities revealing exponential growths, $W(t) \approx W_{0} e^{\alpha t}, N(t) \approx N_{0} e^{\beta t}$, and $E(t) \approx E_{0} e^{\delta t}$, with rates $\alpha=0.036 \pm 0.001, \beta=$ $0.0304 \pm 0.0003$, and $\delta=0.0330 \pm 0.0002$, where $\alpha \gtrsim$ $\delta \gtrsim \beta$ (units are month ${ }^{-1}$ ). These exponential growths, in turn, determine the scaling relations with the system size $W \propto N^{\alpha / \beta}, E \propto N^{\delta / \beta}$, and $\langle k\rangle \propto N^{\delta / \beta-1}$ [15]. All three rates are, indeed, quite close to each other. This result

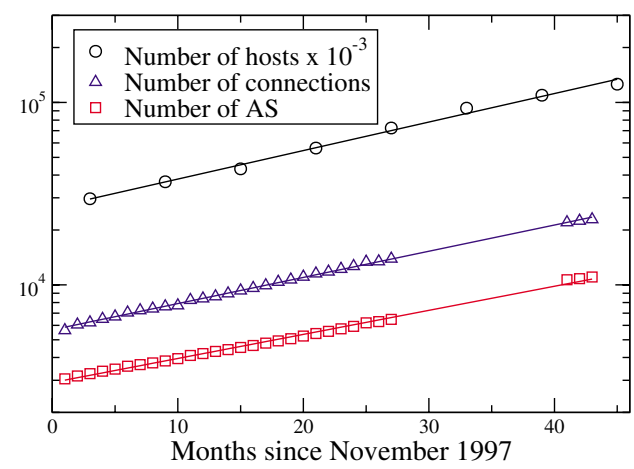

FIG. 1 (color online). Temporal evolution of the number of hosts, autonomous systems, and connections among them from November 1997 to May 2002. Solid lines are the best fit estimates which give the values for the rate growths of $\alpha=0.036 \pm 0.001, \beta=0.0304 \pm 0.0003$, and $\delta=0.0330 \pm$ 0.0002 (units are month ${ }^{-1}$ ). Each point for the number of ASs and connections is an average over one month of daily measurements. Error bars are of the order of the symbol size. 
poses the question of whether these inequalities actually hold or, in contrast, are due to statistical fluctuations. A simple argument convinces us that the inequalities are, actually, the natural answer. There are two mechanisms capable of compensating an increase in the number of users: the creation of new ASs and the creation of new connections by old ASs. When both mechanisms take place simultaneously, the rate of growth of new ASs, $\beta$, must necessarily be smaller than $\alpha$, whereas the rate of growth of the number of connections, $\delta$, must be greater than $\beta$. Any other situation would lead to an imbalance between the number of users and the maximum number of users that the system can manage.

On the basis of these empirical findings, we define our model according to the following rules: (i) At rate $\alpha W(t)$, new users join the system and choose provider $i$ according to some preference function, $\Pi_{i}\left(\left\{\omega_{j}(t)\right\}\right)$, where $\omega_{j}(t), j=$ $1, \ldots, N(t)$, is the number of hosts connected to AS $j$ at time $t$. The function $\Pi_{i}\left(\left\{\omega_{j}(t)\right\}\right)$ is normalized so that $\sum_{i} \Pi_{i}\left(\left\{\omega_{j}(t)\right\}\right)=1$ at any time. (ii) At rate $\beta N(t)$, new ASs join the network with an initial number of users, $\omega_{0}$, randomly withdrawn from the pool of users already attached to existing ASs. Therefore, $\omega_{0}$ can be understood as the minimum number of users required to keep ASs in business. (iii) At rate $\lambda$, each user changes his provider and chooses a new one using the same preference function $\Pi_{i}\left(\left\{\omega_{j}(t)\right\}\right)$. Finally, (iv) each node tries to adapt its number of connections to other nodes according to its present number of users, in an attempt to provide them an adequate access to the Internet. We discuss this last point in the second part of the work. With the above ingredients, in the continuum approximation, the dynamics of single nodes is described by the following differential equation:

$$
\frac{d \omega_{i}}{d t}=(\alpha+\lambda) W(t) \Pi_{i}-\lambda \omega_{i}-\beta \omega_{0} .
$$

The first term on the right-hand side is a creation term accounting for new and old users that choose node $i$ as a provider. The second term represent those users who decide to change their providers and, finally, the last term corresponds to the decrease of users due to the introduction of newly created ASs. To proceed further, we need to specify the preference function $\Pi_{i}\left(\left\{\omega_{j}(t)\right\}\right)$. We assume that, as a result of a competition process, bigger ASs get users more easily than small ones. The simplest function satisfying this condition corresponds to the linear preference, that is,

$$
\Pi_{i}\left(\left\{\omega_{j}(t)\right\}\right)=\frac{\omega_{i}}{W(t)},
$$

where $W(t)=\omega_{0} N_{0} e^{\alpha t}$. In this case, Eq. (1) reads

$$
\frac{d \omega_{i}}{d t}=\alpha \omega_{i}-\beta \omega_{0} .
$$

Notice that reallocation of users (i.e., the $\lambda$ term) has no net effect in the dynamics. After solving Eq. (3), the number of hosts at time $t$ connected to an AS that was introduced at time $t_{i}$ is found to be

$$
\omega_{i}\left(t \mid t_{i}\right)=\frac{\beta}{\alpha} \omega_{0}+\left(1-\frac{\beta}{\alpha}\right) \omega_{0} e^{\alpha\left(t-t_{i}\right)} .
$$

The probability density function of $\omega$ can be calculated in the long time limit as $p(\omega, t)=\beta e^{-\beta t} \int_{0}^{t} e^{\beta t_{i}} \delta(\omega-$ $\left.\omega_{i}\left(t \mid t_{i}\right)\right) d t_{i}$, which leads to

$$
p(\omega, t)=\frac{\tau(1-\tau)^{\tau} \omega_{0}^{\tau}}{\left(\omega-\tau \omega_{0}\right)^{1+\tau}} \Theta\left(\omega_{c}(t)-\omega\right),
$$

where we have defined $\tau \equiv \beta / \alpha$ and the cutoff is given by $\omega_{c}(t) \approx(1-\tau) \omega_{0} e^{\alpha t} \propto W(t)$. Thus, in the long time limit, $p(\omega, t)$ approaches a stationary distribution with an increasing cutoff. In the case of the Internet, $\alpha \gtrsim \beta$, which implies an exponent smaller but close to 2 . A similar result was obtained in [7] using a different approach.

The key point in what follows is how to relate the number of users attached to an AS with its degree. Our basic assumption is that vertices are continuously adapting their bandwidth to the number of users they have. However, once an AS decides to increase its bandwidth it has to find a peer who, at the same time, wants to increase its bandwidth as well. The reason is that connection costs among ASs must be assumed by both peers. This fact differs from other growing models in which vertices do not ask target vertices if they really want to form those connections. Our model is, then, to be thought of as a coupling between a competition process for resources and adaptation of vertices to their current situation, with the constraint that connections are formed only between active nodes. Let $b_{i}\left(t \mid t_{i}\right)$ be the total bandwidth of an AS at time $t$ given that it was introduced at time $t_{i}$. This quantity can include single connections with other ASs, i.e., the topological degree $k$, but it also accounts for connections which have higher capacity. This is equivalent to say that the network is, in fact, weighted and $b_{i}$ is the weighted degree. To simplify the model we consider that bandwidth is discretized in such a way that single connections with high capacity are equivalent to multiple connections between the same ASs. Then, when a pair of ASs agrees to increase their mutual connectivity the connection is newly formed if they were not previously connected or, if they were, their mutual bandwidth increases by one unit. Now, we assume that, at time $t$, each AS adapts its total bandwidth proportionally to its number of users. We can write

$$
b_{i}\left(t \mid t_{i}\right)=1+a(t)\left(\omega_{i}\left(t \mid t_{i}\right)-\omega_{0}\right) .
$$

Summing Eq. (6) for all nodes we get $a(t) \approx \frac{2 B(t)}{W(t)}$, where $B(t)$ is the total bandwidth of the network. $B(t)$ is, obviously, an upper bound to the total number of edges of the network. This suggests that $B(t)$ grows according to $B(t)=$ $B_{0} e^{\delta^{\prime} t}$. As the number of users grows, the global traffic of the Internet also grows, which means that ASs do not only adapt their bandwidth to their number of users but to the global traffic of the network. Therefore, $a(t)$ must be an increasing function of $t$, which, in turn, implies that $\delta^{\prime}>\alpha$ 
and, thus, $\delta^{\prime}>\delta$. As a consequence, the network must necessarily contain multiple connections. This can be explicitly seen by inspecting the scaling of the maximum bandwidth, which reads $b_{c}(t) \propto N(t)^{\delta^{\prime} / \beta}$, that is, faster than $N(t)$. We then propose that degree and bandwidth are related, in a statistical sense, through the following scaling relation:

$$
k\left(t \mid t_{i}\right)=\left[b\left(t \mid t_{i}\right)\right]^{\mu}, \quad \mu<1,
$$

which implies that all nodes can form multiple connections, regardless of their size. This scaling behavior has recently been observed in other weighted networks $[16,17]$. The superlinear behavior of $b_{c}(t)$, combined with this scaling relation, ensures that rich ASs will connect to a macroscopic portion of the system, so that the maximum degree scales linearly with the system size [18]. This sets the scaling exponent to $\mu=\beta / \delta^{\prime}$. All four growth rates in the model are not independent but can be related by exploring the interplay between bandwidth, connectivity, and traffic of the network. Summing Eq. (7) for all vertices, the scaling of the total number of connections is $E(t) \propto N(t)^{2-\alpha / \delta^{\prime}}$, which leads to $\delta^{\prime}=\alpha \beta /(2 \beta-$ $\delta$ ). Combining this relation with Eqs. (5)-(7), the degree distribution reads

$$
P(k) \approx \frac{\tau(1-\tau)^{\tau}\left[\omega_{0} a(t)\right]^{\tau}}{\mu} \frac{1}{k^{\gamma}} \Theta\left(k_{c}(t)-k\right)
$$

for $k \gg 1$, where the exponent $\gamma$ takes the value

$$
\gamma=1+\frac{1}{2-\delta / \beta} .
$$

Strikingly, the exponent $\gamma$ has lost any direct dependence on $\alpha$ becoming a function of the ratio $\delta / \beta$. Using the empirical values for $\beta$ and $\delta$, the predicted exponent is $\gamma=2.2 \pm 0.1$, in excellent agreement with the values reported in the literature $[11,12]$. Of course, this does not mean that the exponent $\gamma$ is independent of $\alpha$, since both, $\beta$ and $\delta$, may depend on the growth of the number of users. However, the exact dependence between these exponents could be derived only by modeling the balance between demand and supply in the system.

So far, we have been mainly interested in the degree distribution of the AS map but not in the specific way in which the network is formed. To fill this gap we have performed numerical simulations that generate network topologies in nice agreement with real measures of the Internet. We also consider a realistic geographical deployment of ASs and the physical distance among them to take into account connection costs [10]. Our algorithm, following the lines of the model, works in four steps:

(1) At iteration $t, \Delta W(t)=\omega_{0} N_{0}\left(e^{\alpha t}-e^{\alpha(t-1)}\right)$ users join the network and choose a provider among the existing nodes using the preference rule Eq. (2).

(2) $\Delta N(t)=N_{0}\left(e^{\beta t}-e^{\beta(t-1)}\right)$ new ASs are introduced with $\omega_{0}$ users each, those being randomly withdrawn from already existing ASs. Newly created ASs are located in a two dimensional plane following a fractal set of dimension $D_{f}=1.5$ [10].

(3) Each AS evaluates its increase of bandwidth, $\Delta b_{i}\left(t \mid t_{i}\right)$, according to Eq. (6).

(4) A pair of nodes, $(i, j)$, is chosen with probability proportional to $\Delta b_{i}\left(t \mid t_{i}\right)$ and $\Delta b_{j}\left(t \mid t_{j}\right)$ respectively, and, whenever they both need to increase their bandwidth, they form a connection with probability $D\left(d_{i j}, \omega_{i}, \omega_{j}\right)$. This function takes into consideration that, due to connection costs, physical links over long distances are unlikely to be created by small peers. Once the first connection has been formed, they create a new connection with probability $r$, whenever they still need to increase their bandwidth. This step is repeated until all nodes have the desired bandwidth.

It is important to stress the fact that nodes must be chosen with probability proportional to their increase in bandwidth at each step. The reason is that those nodes that need a high bandwidth increase will be more active when looking for partners to whom form connections. Another important point is the role of the parameter $r$. This parameter takes into account the balance between the costs of forming connections with new peers and the need for diversification in the number of partners. The effect of $r$ in the network topology is to tune the average degree and the clustering coefficient by creating more multiple connections. The exponent $\gamma$ is unaffected except in the limiting case $r \rightarrow 1$. In this situation, big peers will create a huge amount of multiple connections among them, reducing, thus, the maximum degree of the network. Finally, we choose an exponential form for the distance probability function $D\left(d_{i j}, \omega_{i}, \omega_{j}\right)=e^{-d_{i j} / d_{c}\left(\omega_{i}, \omega_{j}\right)}$, where $d_{c}\left(\omega_{i}, \omega_{j}\right)=\omega_{i} \omega_{j} / \kappa W(t)$ and $\kappa$ is a cost function of number of users per unit distance, depending on the maximum distance of the fractal set. All simulations are performed using $\omega_{0}=5000, N_{0}=2, B_{0}=1, \alpha=0.035$, $\beta=0.03$, and $\delta^{\prime}=0.04$, and the final size of the networks is $N \approx 11000$. Simulations will be compared to the $\mathrm{AS}^{+}$ extended map recorded on May 2001, as reported in [19] that offers a better picture of the actual map.

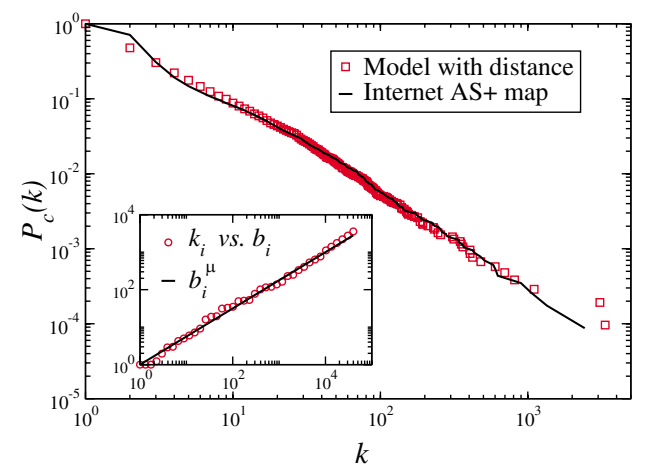

FIG. 2 (color online). Cumulative degree distribution $\left[P_{c}(k)=\right.$ $\left.\sum_{k} P\left(k^{\prime}\right)\right]$ for the extended AS map compared to simulations of the model, $r=0.8$. Inset: simulation results of the AS's degree as a function of the AS's bandwidth. The solid line stands for the scaling relation Eq. (7) with $\mu=\beta / \delta^{\prime}=0.75$. 

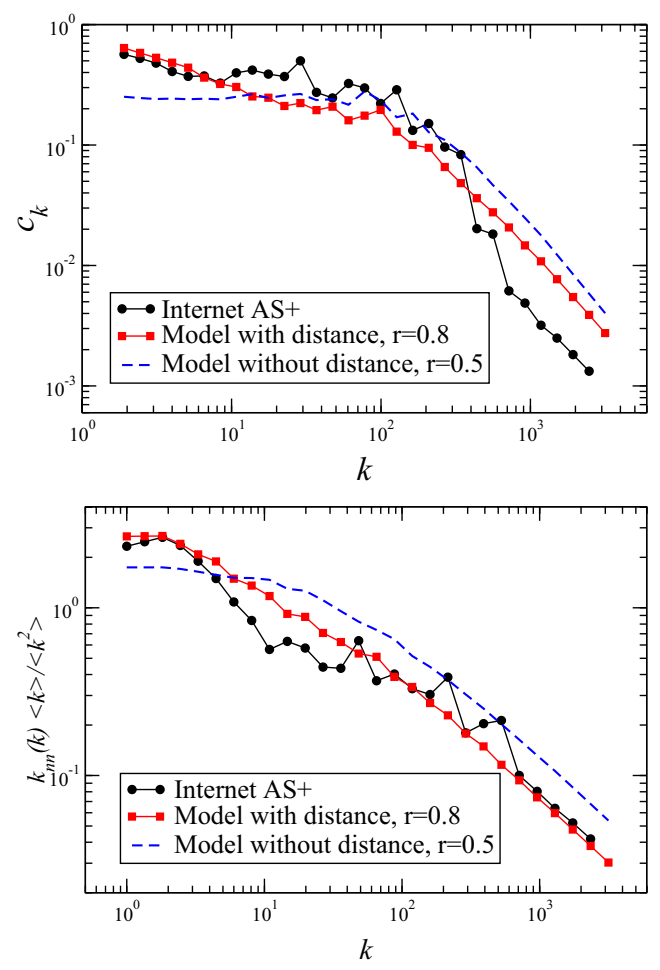

FIG. 3 (color online). Clustering coefficient, $c_{k}$ (top) and normalized average nearest neighbors degree, $\bar{k}_{n n}(k)\langle k\rangle /\left\langle k^{2}\right\rangle$ (bottom), as functions of the node's degree for the extended autonomous system map (circles) and for the model with and without distance constraints (red squares and dashed line, respectively).

Figure 2 shows simulation results for the cumulative degree distribution, in nice agreement to that measured for the $\mathrm{AS}^{+}$map. The inset exhibits simulation results of the AS's degree as a function of the AS's bandwidth, confirming the scaling ansatz Eq. (7). Clustering coefficient and average nearest neighbors degree are shown in Fig. 3. The dashed lines result from the model without distance constraints, whereas the squares correspond to the model with distance constraints. Interestingly, the high level of clustering coming out from the model arises as a consequence of the pattern followed to attach nodes, so that only those ASs willing for new connections will link. As can be observed in the figures, distance constraints introduce a disassortative component by inhibiting connections between small ASs so that the hierarchical structure of the real network is better reproduced.

In summary, we have presented a novel growth network model, where both nodes and links are weighted. The dynamical evolution is driven by two key mechanisms, competition and adaptation, which may be relevant in other self-organizing systems. We propose a scaling law to link the degree of a node with its bandwidth, which allows us to obtain the degree exponent $\gamma$ as a function of the measurable quantities $\beta$ and $\delta$. Two and three point correlations are nicely reproduced, as well as the loop structure, recently analyzed in [20]. Beyond technical details, the
Internet is a paradigmatic example in the realm of complexity theory. Our model provides deeper insights on its evolution, which can shed light on the understanding of the dynamical processes leading to network formation in a more general framework.

We acknowledge R. Pastor-Satorras and A. Arenas for valuable comments and suggestions. This work has been partially supported by DGES of the Spanish government, Grant No. BFM2003-08258 and by EC-FET Open Project No. COSIN IST-2001-33555. M. B. acknowledges financial support from the MCyT (Spain).

[1] R. Pastor-Satorras and A. Vespignani, Evolution and Structure of the Internet. A Statistical Physics Approach (Cambridge University Press, Cambridge, 2004).

[2] S. N. Dorogovtsev and J.F.F. Mendes, Evolution of Networks: From Biological Nets to the Internet and $W W W$ (Oxford University Press, Oxford, 2003).

[3] A.-L. Barabási and R. Albert, Science 286, 509 (1999).

[4] B. A. Huberman and L. A. Adamic, Nature (London) 401, 131 (1999).

[5] K.-I. Goh, B. Kahng, and D. Kim, Phys. Rev. Lett. 88, 108701 (2002).

[6] A. Capocci, G. Caldarelli, R. Marchetti, and L. Pietronero, Phys. Rev. E 64, 035105 (2001).

[7] M. Fayed, P. Krapivsky, J. W. Byers, M. Crovella, D. Finkel, and S. Redner, Comput. Commun. Rev. 33, 41 (2003).

[8] A. Medina, I. Matta, and J. Byers, Comput. Commun. Rev. 30, 18 (2000).

[9] S. Zhou, and R. J. Mondragon, cs.NI/0402011.

[10] S. H. Yook, H. Jeong, and A.-L. Barabási, Proc. Nat. Acad. Sci. U.S.A. 99, 13382 (2002).

[11] M. Faloutsos, P. Faloutsos, and C. Faloutsos, Comput. Commun. Rev. 29, 251 (1999).

[12] R. Pastor-Satorras, A. Vázquez, and A. Vespignani, Phys. Rev. Lett. 87, 258701 (2001); A. Vázquez, R. PastorSatorras, and A. Vespignani, Phys. Rev. E 65, 066130 (2002).

[13] Data from the Hobbes's Internet Timeline http://www.zakon.org/robert/internet/timeline/.

[14] Available data correspond to daily measurements from November 1997 to January 2000 and three months of 2002. http://moat.nlanr.net/Routing/rawdata/

[15] S. N. Dorogovtsev and J.F.F. Mendes, Phys. Rev. E 63, 025101 (2001).

[16] A. Barrat, M. Barthélemy, R. Pastor-Satorras, and A. Vespignani, Proc. Natl. Acad. Sci. U.S.A. 101, 3747 (2004).

[17] A. Barrat, M. Barthélemy, and A. Vespignani, Phys. Rev. Lett. 92, 228701 (2004).

[18] Empirical measurements made in [5] showed such linear scaling in the AS with the largest degree.

[19] C. Qian, H. Chang, R. Govindan, S. Jamin, S. Shenker, and W. Willinger, in Proceedings of IEEE INFOCOM, New York, 2002 (IEEE, Piscataway, NJ, 2002), Vol. 2, p. 608.

[20] G. Bianconi, G. Caldarelli, and A. Capocci, cond-mat/ 0408349; (private communication). 